\title{
Multi-Level Barriers to LTBI Treatment: A Research Note
}

\author{
Linda Hill · Elaine Blumberg · Carol Sipan · \\ Katharine Schmitz · Joshua West · Norma Kelley • \\ Melbourne Hovell
}

Published online: 16 December 2008

(C) The Author(s) 2008. This article is published with open access at Springerlink.com

\begin{abstract}
Background This study describes the barriers to effective and timely LTBI treatment encountered in a research study on INH adherence in Latino adolescents. Method Participant study logs were reviewed, results of continuing medical education pretests for medical providers were examined, and participating medical facilities were contacted in order to construct a profile of multilevel barriers to LTBI treatment. Results A total of 285 TST positive Latino (96\%) high school students were
\end{abstract}

\author{
L. Hill ( $₫)$ \\ Department of Family and Preventive Medicine, University \\ of California, San Diego, 9500 Gilman Dr., MS0811, La Jolla, \\ CA 92037-0811, USA \\ e-mail: lhillbaird@aol.com \\ E. Blumberg - C. Sipan - K. Schmitz · J. West · N. Kelley · \\ M. Hovell \\ Center for Behavioral Epidemiology and Community Health \\ (C-BEACH), Graduate School of Public Health, San Diego State \\ University, 9245 Sky Park Court, Ste 230, San Diego, CA 92123, \\ USA \\ E. Blumberg \\ e-mail: eblumberg@projects.sdsu.edu \\ C. Sipan \\ e-mail: csipan@projects.sdsu.edu \\ K. Schmitz \\ e-mail: kschmitz@projects.sdsu.edu \\ J. West \\ e-mail: jwest@projects.sdsu.edu \\ N. Kelley \\ e-mail: nkelley@projects.sdsu.edu \\ M. Hovell \\ e-mail: mhovell@projects.sdsu.edu
}

recruited into the trial. We encountered a lack of understanding of the gravity of tuberculosis infection among both the public and providers of health care. Parents and adolescents cited competing priorities, transportation problems and financial constraints as reasons for non-compliance. Discussion Improved education of the public and physicians is needed regarding the gravity of the disease and the value of treatment, as well as public and financial support for LTBI treatment by both the government and the medical community.

Keywords Latent tuberculosis infection - Compliance · Latino adolescents · Barriers to treatment

\section{Background}

Tuberculosis (TB) continues to be a worldwide cause of morbidity and mortality, with an estimated 8.8 million new cases in 2005 [1]. The emergence of multi-drug resistant TB (MDRTB) and extremely drug resistant TB (XDRTB), and the association of TB with HIV have kept TB in the forefront of public health concerns [2]. Tuberculosis infection (LTBI) is present in one-third of the world's population [3]. LTBI, from which the majority of disease is derived, proceeds to active disease in approximately $4-13 \%$ of TB-infected HIVnegative individuals within 5 years [4], and at a rate of $10 \%$ per year in TB-infected HIV-positive individuals [5]. Approximately 5\% (15 million individuals) of the U.S. population has LTBI; active TB was reported in approximately 14,000 individuals in 2005 , and is most prevalent in low income and immigrant populations [6].

The Advisory Council for the Elimination of Tuberculosis (ACET) recommends expanded "testing and treatment of LTBI among targeted populations" in the efforts to meet TB 
elimination goals [7]. The most commonly recommended treatment for LTBI in the US is a 9 month course of isoniazid (INH), which is over $90 \%$ efficacious in preventing progression, even in individuals with variable compliance [8]. Treatment of children and adolescents is felt to be particularly important because of their increased risk of progression to active TB, expected greater number of years of risk, as well as the safety of treatment $[8,9]$.

Treatment of active TB has been compromised by poor patient compliance, which is well documented. Directly Observed Therapy (DOT) is now the community standard for active disease treatment [10-16]. Compliance with LTBI treatment is also problematic; in the US, completion of even a minimum of 6 months of LTBI treatment by infected adolescents is estimated at only $33-74 \%[9,17-$ 19]. The Healthy People 2010 treatment goal is $85 \%$ [20]. The purpose of this paper is to describe the barriers to effective and timely LTBI treatment encountered in a research study on isoniazid adherence in adolescents.

\section{Methods}

This report derives from a 5-year NIH-funded study designed to evaluate the effectiveness of peer counseling combined with parent training to increase isoniazid adherence in adolescents with LTBI. For the present report, participant study logs (which included records of all participant activities) were reviewed, results of continuing medical education (CME) course pre-tests for medical providers were examined, and participating medical facilities were contacted in order to construct a profile of the multi-level barriers to LTBI treatment. Barriers related to participants, parents, medical providers, and funding were examined.

Seven high schools in San Diego County, with student populations between 13 and 21, were targeted for screening. All of these schools are located within 15 miles of the Mexican border and thus have a high percentage of Latino and immigrant youth. Students that screened positive $(>10 \mathrm{~mm})$ and their parents were invited to attend informational sessions on LTBI, which were provided at their child's school, and included education about TB, treatment, transmission, and the research project.

Students found to be TST positive and one parent were offered enrollment in the adherence trial; confirmation was obtained before screening and before enrollment that they had not been previously treated for TB or LTBI. Bilingual, culturally competent staff completed all of the participant recruitment. All TST positive participants were offered free chest X-rays (CXRs), help finding a medical home if needed, and help scheduling and attending their first medical appointment. The purpose of the CXR was clearly explained to the participating parent and student. One parent and participants were administered a baseline questionnaire after enrollment in the study.

Prior to a three-lecture CME course, clinicians completed a 40-question pre-test to assess LTBI knowledge. The questions included topics on skin tests, medication and duration of treatment, dosages, side effects, and co-infection with HIV. For the purposes of the CME testing, the CME content, and this study, the following LTBI treatment protocols were recommended (based on the CDC recommendations for LTBI [21]): 9 months of isoniazid, 10 $20 \mathrm{mg} / \mathrm{kg}$ up to $300 \mathrm{mg} / \mathrm{day}$; baseline screening by history for liver disease and alcohol use; baseline liver function tests only for those patients with a positive history of liver disease or alcohol abuse; monthly visits for review of side effects with face-to-face encounters; only 1 month of medication given each visit; baseline patient education of side effects and instructions to return sooner than 1 month if side effects develop; 9 months of isoniazid must be taken within a 12-month period (i.e., short gaps do not require restarting medications); B-6 supplementation when dietary intake is not expected to meet the requirements.

Prior to the completion of the intervention, five of the participating health centers were contacted to ascertain protocols and practice of LTBI treatment. The health centers reflected the care received by the participants: one private practice, two community health centers, one military health center (for dependents) and the County LTBI clinic. A survey was done in person by a FIESTA staff member (a registered nurse and MPH student) regarding LTBI medications, dosages, length of treatment, follow-up, and lab testing.

This study was approved by the Committee on Protection of Human Subjects of San Diego State University.

\section{Results}

Participants

Of the 285 TST positive youths recruited into the trial, the mean age was 15.9 years $( \pm 1.2)$ (Table 1$)$. Fifty percent were females and $49 \%$ had no health insurance. Forty-five percent were foreign born and $96 \%$ were Latino. The majority of the participants (90\%) spoke two languages, Spanish and English, with Spanish being the primary language for about $85 \%$. Sixty-two percent were considered to be bicultural using the Marin \& Gamba acculturation scale [22].

Parents

The majority of parents who participated were female (86\%), with most (80\%) being the participant's Mother 
Table 1 Baseline characteristics and knowledge of participants (adolescent and parent)

\begin{tabular}{lll}
\hline Characteristic & Adolescents & Parents \\
\hline Sample size & 285 & 285 \\
$\%$ female & $50 \%$ & $86 \%$ (80\% mother) \\
$\%$ Latino & $96 \%$ & $96 \%$ \\
$\%$ foreign born & $45 \%$ & $93 \%$ \\
Spanish primary language & $85 \%$ & $89 \%$ \\
$\%$ with no health insurance & $41.8 \%$ & $45 \%$ \\
Median household income & NA & $\$ 20,000-29,000$ \\
& $\%$ of respondents who selected the incorrect \\
& response or 'don't know' & 7.1 \\
Did not know that TB can be detected by a skin test & 22.5 & 3.5 \\
Did not know that LTBI treatment reduces risk of TB & 15.1 & 15.8 \\
Did not feel that TB can be cured & 32.6 & NA \\
Not worried when they found out they had a positive TST & 26.7 & \\
\hline
\end{tabular}

(Table 1). The mean age of the participating parent was 42.5 years $( \pm 7.8)$ and 44.0 years $( \pm 7.8)$ for the other parent. The mean years of education for both parents was over nine years (participating parent: $M=9.5, \pm 3.6$; other parent: $M=9.6, \pm 3.9)$. Ninety-six percent of both parents were Latinos, with $89 \%$ of the participating parents born in Mexico (93\% were foreign born) and $87 \%$ of the other parents born in Mexico (90\% were foreign born). Sixty-six percent of the participating parents spoke only one language with the primary language being Spanish for $83 \%$, while $60 \%$ of the other parent spoke two languages, again with Spanish being the primary language for the majority, $89 \%$. The median income for the previous year was $\$<10,000$ for the participating parent and $\$ 20,000-29,000$ for the other parent, with the median for the household being \$20,000-29,000. Forty-five percent reported not having any medical insurance.

\section{Participant Barriers}

A diagnosis of LTBI involves a positive TST and a negative CXR. X-rays are required to rule out active TB. Participants were encouraged to obtain a CXR as soon as possible, and staff maintained constant contact to monitor their progress to this end. Participants were contacted four to five times a week for a month, and then weekly until they were on medications (isoniazid). Assistance was provided as necessary. Many participants required prompting and assistance to obtain baseline X-rays, initiate treatment, and maintain treatment. About $46 \%$ of participants required assistance in receiving a CXR, requiring a mean of 1.5 contacts (range 0-14) in the form of telephone calls and/or home visits by project staff. Almost $19 \%$ required a home visit(s) to schedule the initial medical visit to get started on isoniazid (mean of 0.7 , range $0-23$ ) and $58 \%$ required phone call(s) (mean of 3.2, range 0-39). Home visits and phone calls were used to provide additional patient/parent education and to facilitate compliance with clinic appointments to initiate isoniazid.

Once appointments were scheduled, compliance with these medical visits was also a problem. Thirty-four participants $(12 \%)$ missed one or more initial clinic appointments (range 0-6), and 15 (5\%) required transportation by project staff. Continuing care was also an issue, with ongoing prompting required to enhance compliance with the follow-up clinic appointments.

Participants reported a general lack of knowledge of LTBI and TB at the initial interview. Twenty-two percent did not know that TB could be detected by a skin test; $15 \%$ did not know that LTBI treatment reduces the risk of TB, and $32 \%$ did not know that TB could be cured.

In addition to the routine continuity visits, some participants reported symptoms compatible with isoniazid side effects to our staff during intervention and measures visits, which required study staff to facilitate getting participants to their medical providers for unforeseen visits. Although no true isoniazid side effects were detected by providers in this study, the expenses for these visits were outside of the routine LTBI care, and often involved blood tests, which lead to financial issues for the participants, and difficulty in enforcing compliance with these visits.

\section{Parental Barriers}

The baseline questionnaire revealed a lack of parental knowledge regarding LTBI and LTBI treatment. Twenty percent of the participating parents did not know that LTBI could be detected by a skin test and $13 \%$ did not know that completing LTBI treatment greatly reduces risk of TB. Thirty percent of parents did not feel that TB could be cured, and $26 \%$ were 'not worried when they found out their child had a positive TST.' Forty percent of parents did 
not think that positive TST reflected being 'infected with TB bacteria at some time in the past,' while $14 \%$ thought it was from 'receiving BCG vaccine as a child,' and 19\% attributed it to 'scratching the site.'

Parents' work conflicts and lack of transportation contributed to the difficulties in scheduling appointments for the CXR and isoniazid. This barrier was solved for those adolescents with a means of independent transportation through obtaining parental consent forms that permitted LTBI treatment without their presence. Parents frequently attributed lack of attendance to medical visits to costs to see a provider, even the modest charges of community health centers.

One parent's concerns about potential side effects in their child prompted cessation of LTBI treatment and dropping out of the study.

\section{Provider-Related Barriers}

The providers in the participating San Diego health centers were given the opportunity to obtain $3 \mathrm{~h}$ of CME credit for attending on-site LTBI in-services. Eighty-five physicians and mid-level practitioners were offered CME. Of these, 24 (28\%) participated, yet just $17(20 \%)$ providers took the CME pre-test required to obtain CME credit. Only 13 (77\%) of the 17 completed pre-tests received a passing grade $(60 \%$ correct). The post-tests were completed by 9 of the 17 providers, with $78 \%$ receiving a passing grade. During the in-services, we also encountered resistance to the CDC recommendations for monthly visits to evaluate persons treated with isoniazid for side effects. Some providers stated that 'their patients would not return monthly,' and, for that reason, they planned to continue prescribing/ dispensing 2-3 months or more of medication at a time.

Five of the participating health centers completed the survey to assess their LTBI standards of care. The survey results were compared with the CDC's guidelines for LTBI treatment. Respondents for the participating health centers included one medical director (physician), one staff physician, one RN, one public health nurse, and one medical assistant. Four of the five clinics correctly identified the dosage of isoniazid for daily regimes, with four of the clinics correctly identifying $300 \mathrm{mg}$ as the maximum dosage for daily regimes. Three of the clinics correctly cited 9 months of treatment as the correct length of treatment for daily treatment with isoniazid; three also had protocols to see patients monthly. The other two clinics saw patients at initial visit only (one) and initial visit and final visit (one).

Of the participants who were given only 1 month of isoniazid at a time, 120/156 (77\%) came back for the 1 month follow up, and 140/156 (90\%) came back for at least one more visit. However, of the participants who were given more than 1 month, only $9 / 19$ (47\%) came back before their prescription ran out, and 13/19 (68\%) ever came back. For those participants whose charts did not indicate the number of pills given, only $17 / 48(35 \%)$ ever had another visit.

\section{Funding-Related Barriers}

Until 2004, the County of San Diego was able to provide public funded care for LTBI, either through direct services, or through subcontracts with the community health clinic (CHC) system. However, due to shrinking public health funds in general as well as those for TB control, CDC's priorities narrowed to active tuberculosis treatment and contact tracing and care. The County is now only able to provide limited direct LTBI care, and does no subcontracting. Per parental reports, even the modest charges of the participating CHCs for LTBI treatment were prohibitive for some participants. Similarly, providing care at discounted rates that families could afford was prohibitive for many of the community clinics to afford.

\section{Participant Feedback About Clinical Care}

Participants provided study staff feedback on their contacts with physicians when attempting to get started on isoniazid, reflecting misinformation and mistreatment. This included inappropriate dosing, not scheduling monthly visits, inappropriate length of treatment regimens, and inappropriate staff and/or methods of obtaining medical histories of possible side effects of isoniazid. Some participants seeing providers in San Diego reported that they were told: "Adolescents don't need treatment for LTBI", "Only 6 months of treatment is needed," "Isoniazid is too toxic to use in adolescents." Several participants seeing providers in Tijuana heard: "The positive TST in Mexicans is from BCG, and your child doesn't need treatment" and "Don't take medicine, just get a CXR every year." This type of misinformation contributed to refusal to enroll in the study by at least 16 potential participants.

Additional problems were reported by parents and participants regarding obtaining refills. In some cases, families were told that medication was ready, but when they arrived at the facility to pick it up, there was no prescription. Patients attending facilities using a mail system, where medication refills of isoniazid were mailed to the patient's home, reported never receiving the refills. Patients reported sometimes being asked about side effects by a nurse, a medical assistant, a receptionist, or not at all. This questioning sometimes took place in person, and sometimes by phone. 


\section{Discussion}

Successful treatment of LTBI in adolescents requires targeted screening, appropriate initiation of treatment, and compliance with the entire treatment regimen [18, 23, 24]. The results of this study are consistent with barriers identified in other studies, including a serious lack of understanding of the gravity of LTBI amongst both the public and providers of health care [25, 26]. Generally, providers demonstrated a lack of basic knowledge of TB on written tests and in practice. Those providers who did not follow CDC guidelines on number of pills to give at a time (1 month) were less likely to see their patients before the medication was finished, and less likely to ever see the patients, with implications for both compliance and screening for side effects.

This is also consistent with other studies demonstrating gaps between current CDC guidelines for LTBI treatment, and physicians' attitudes and practice in the community [27]. This included misinformation regarding treatment of LTBI in patients TST positive, who had received BCG, despite CDC recommendations to ignore the BCG history [21]. Furthermore, we encountered a lack of interest from the parents and adolescents as evidenced by the difficulty in getting adolescents screened and enrolled. The efforts expended to get these adolescents into care, with multiple attempts required, are completely unrealistic for nonresearch settings in absence of substantial financial support not now available.

One practice we put into place for study participants was to request that parents sign consent forms for their child's LTBI treatment without the parents being physically present at the time of the visit. This is an option that may be useful for older adolescents in LTBI care in community settings. System-wide solutions to barriers include streamlining LTBI follow-up visits, using dedicated LTBI staff, and having consistent methodologies for screening for side effects and refilling medicine. These might have eliminated much of the frustration reported by our participants in obtaining refills, and the frustrations clinic personnel encountered when patients showed for TB care but were not in the appointment system or when patients did not show when they were scheduled.

National funding for LTBI treatment increased after the 2000 IOM report Ending Neglect [28], however the five million dollars of designated funding was only available for 5 years (Dr. K. Moser, personal communication). The CDC outlined recommendations for LTBI activities in their "CDC's Response to ending neglect: The elimination of tuberculosis in the US 2002" [29]. However, after 2004, the Division of TB Elimination at the CDC prioritized other TB control activities, including active disease, contact tracing, research, genotyping, education centers, and others.
Currently no funding from the CDC specifically targets screening and treatment for LTBI in the US. Yet, it has been estimated that to eliminate active tuberculosis infection from a country the LTBI rates must be reduced to $1 /$ million population; current estimates based on NHANES data show 4.3/million civilian, non-institutionalized population as infected [30, 31]. Significant efforts will be required to achieve a $75 \%$ reduction in the prevalence of untreated LTBI in the US.

This report has implications for the development of strategies to promote LTBI screening and treatment. First, the public needs to have a greater understanding of the gravity of LTBI, and the implications for the future health of infected individuals. Since a number of parents felt that their child's positive TST was due to receipt of BCG, the availability of more accurate testing for LTBI, such as Quantiferon, may be useful to overcome this barrier. This is especially important in patients from Mexico and other countries where BCG is commonly used.

Second, physicians need ongoing training to understand and implement policies for screening and appropriate treatment. Third, public funds are necessary to treat the medically indigent infected with LTBI in order to improve compliance and protect the whole population from TB infection. Such treatment is necessary to reduce the ongoing conversion to active TB and the continuing episodic epidemic outbreaks. With increasing frequency and volume of travel world-wide, such outbreaks will become more frequent and the spread across nations may take only hours to occur. Effecting prevention of active TB by treating those with LTBI remains the best means of reducing the incidence of active disease.

Our findings suggest that the traditional clinical practice procedures do not work well for TB prevention among adolescents. Efforts should be undertaken to modify traditional patient provider examinations and counseling to make treatment completion more likely while ensuring satisfactory safety. This could be achieved by instituting changes in the delivery of care for TB prevention. Among these changes might be use of an "advice nurse" who could track patients for follow-up contact/visits and new prescriptions. This might involve face-to-face meetings on some frequency less than monthly and telephone or email contact to obtain information about possible side effects and the need for more complete examination. Use of incentives for preventive care should be considered key for completing all prevention services and they can be justified on the basis of protecting the public as much or more than the patient. Providers should be compensated for TB prevention services and held responsible for adhering to CDC guidelines, even if such guidelines warrant greater flexibility to match both patient and provider needs for convenient delivery of care. Experts in public health, 
medical care and TB prevention in particular should contribute to refinements in current guidelines that allow tailoring of medical monitoring that includes telemedicine procedures and use of physician assistants to make the overarching system more practical, effective and safe.

This report is limited by the observational nature of the data. Due to the large Latino representation in this study, we cannot generalize to other communities. Nevertheless, we have no reason to believe that the findings are not representative of the attitudes and behavior of other parents and physicians of LTBI infected adolescents in communities with similar demographics and with similar short falls in public support for screening and preventive treatments for LTBI.

Tuberculosis continues to be a worldwide cause of morbidity and mortality. The pool of infected individuals with LTBI must be treated with appropriate therapeutics at the recommended length of time to reduce the rates of reactivation. Improved education of the public and physicians is needed regarding the gravity of the disease and the value of treatment, as well as public and financial support for LTBI treatment by both the government and the medical community.

Acknowledgements This study was supported by the National Heart, Lung, and Blood Institute, National Institutes of Health, Grant \#5RO1 HL068595 awarded to Dr. Mel Hovell and the San Diego State University Research Foundation and by intramural support from $\mathrm{CBEACH}$.

Open Access This article is distributed under the terms of the Creative Commons Attribution Noncommercial License which permits any noncommercial use, distribution, and reproduction in any medium, provided the original author(s) and source are credited.

\section{References}

1. World Health Organization. Tuberculosis fact sheet. March 2007. http://www.who.int/mediacentre/factsheets/fs104/en/. Accessed 22 July 2008

2. World Health Organization. Strategic framework to decrease the burden of TB/HIV. Geneva, World Health Organization, 2002; WHO/CDS/TB/2002.296, WHO/HIV_AIDS/2002.2.

3. World Health Organization. Tuberculosis facts 2007. http://www. who.int/tb/publications/2007/factsheet_2007.pdf. Accessed 22 July 2008.

4. Vynnychy E, Fine PE. The natural history of tuberculosis: the implications of age-dependent risks of disease and the role of reinfection. Epidemiol Infect. 1997;119:183-201.

5. Selwyn PA, Hartel D, et al. A prospective study of the risk of tuberculosis among intravenous drug users with human immunodeficiency virus infection. N Engl J Med. 1989;320:545-50.

6. Centers for Disease Control and Prevention. Reported tuberculosis in the US. Atlanta, GA: US DHHS, CDC; Sept 2006.

7. Centers for Disease Control and Prevention. Tuberculosis elimination revisited: obstacles, opportunities, and a renewed commitment-advisory council for the elimination of tuberculosis (ACET). MMWR. 1999;48(RR09):1-13.
8. Pediatric Tuberculosis Collaborative Group. Targeted tuberculin skin testing and treatment of latent tuberculosis infection in children and adolescents. Pediatrics. 2004;114(4):1175-201.

9. Morisky D, Ebin V, et al. Assessment of tuberculosis treatment completion in an ethnically diverse population using two data sources. Eval Health Prof. 2003;26(1):43-58.

10. Addington WW. Patient compliance: the most serious remaining problem in the control of tuberculosis in the United States. Chest. 1979;76:741-3.

11. Fox W. Compliance of patients and physicians: experience and lessons from tuberculosis-I. BMJ. 1983;287:33-5.

12. Sumartojo E. When tuberculosis treatment fails: a social behavioral account of patient adherence. Am Rev Respir Dis. 1993; 147:1311-20.

13. Mullen PD. Compliance becomes concordance. BMJ. 1997; 314:691-2.

14. Centers for Disease Control and Prevention. Approaches to improving adherence to antituberculosis therapy. MMWR 1993; 42:74-5, 81 .

15. Sbarbaro JA, Sbarbaro JB. Compliance and supervision of chemotherapy of tuberculosis. Sem Respir Infect. 1994;9:120-7.

16. Volmink J, Garner P. Strategies for promoting completion of treatment in patients with tuberculosis. In: Garner P, Gelband H, Olliaro P, Salinas R, Volmink J, Wilkinson D, editors. Infectious diseases module of the Cochrane database of systematic reviews [updated 15 April 1997]. Oxford: Update Software; 1997 (available in the Cochrane Library).

17. Starr M, Sawyer S, et al. A novel approach to monitoring adherence to preventive therapy for tuberculosis in adolescence. $\mathbf{J}$ Pediatr Child Health. 1999;35(4):350-4.

18. Barry MA, Shirley L, et al. Tuberculosis infection in urban adolescents: results of a school based testing program. AJPH. 1990;80:439-41.

19. Hovell MF, Sipan CL, Blumberg EJ, Hofstetter CR, Slymen D, Friedman L, Moser K, Kelley NJ, Vera AY. Increasing Latino adolescents' adherence to treatment for latent Tuberculosis infection: a controlled trial. AJPH. 2003;93:1871-7.

20. US Department of Health and Human Services. Office of disease prevention and health promotion: healthy people 2010, Vol. 1, 2nd ed. http://www.healthypeople.gov/Document/pdf/Volume1/ 14Immunization.pdf. Accessed 22 July 2008.

21. Centers for Disease Control, Prevention, Guide for Primary Care Providers. Targeted tuberculin testing, treatment of latent tuberculosis infection. Atlanta, GA: DHHS, CDC, National Center for HIV, STD, and TB Prevention, Division of Tuberculosis Elimination; 2005.

22. Kerner JF, Breen N, Tefft MC, Silsby J, Marın G, Gamba RJ. A new measure of acculturation for hispanics: The bidimensional scale for hispanics (BAS). Hisp J Behav Sci. 1996;18(3):297-316.

23. Centers for Disease Control and Prevention. The use of preventive therapy for tuberculosis infection in the United States: recommendations of the Advisory Committee for Elimination of Tuberculosis. MMWR. 1995;30(RR-8):9-12.

24. Ozuah PO, Ozuah TP, Stein RE, Burton W, Mulvihill M. Evaluation of a risk assessment questionnaire used to target tuberculin skin testing in children. JAMA. 2001;285(4):451-3.

25. Shieh FK, Snyder G, Horsburgh CR, Bernardo J, Murphy C, Saukkonen JJ. Predicting non-completion of treatment for latent tuberculosis infection: a prospective survey. Am J Respir Crit Care Med. 2006;174(6):717-21.

26. Jackson M, Harrity S, Hoffman H, Catanzaro A. A survey of health professions students for knowledge, attitudes, and confidence about tuberculosis, 2005. BMC Publ Health. 2007; 7(147):219.

27. Salazar-Schicchi J, Jedlovsky V, Ajayi A, Colson PW, HirschMoverman Y, El-Sadr W. Physician attitudes regarding bacille 
Calmette-Guerin vaccination and treatment of latent tuberculosis infection. Int J Tuberc Lung Dis. 2004;8(12):1443-7.

28. Institute of Medicine. Ending neglect: the elimination of tuberculosis in the United States 2000. Geiter L, editor. Committee on the Elimination of Tuberculosis in the United States, Division of Health Promotion and Disease Prevention. Washigton, DC: National Academy Press.

29. Centers for Disease Control and Prevention. CDC's Response to ending neglect: The elimination of tuberculosis in the US 2002. http://www.cdc.gov/tb/pubs/iom/iomresponse/default.htm, Accessed 22 July 2008.
30. Styblo K. Epidemiology of tuberculosis. Selected papers, vol 24. The Hague, The Netherlands: Royal Netherlands Tuberculosis Association (KNCV); 1991. p. 49-50.

31. Bennett D, Couval JM, Onorato MD, et al. Prevalence of TB infection in the US population 1999-2000. In: Program and abstracts of the American Public Health Association 131st annual meeting; San Francisco, California, November 15-19, 2003. Session 4199. 